\title{
Intramolecular electron diffraction in vibrationally resolved $K$-shell photoionization of methane
}

\author{
Etienne Plésiat, ${ }^{1}$ Luca Argenti, ${ }^{1}$ Edwin Kukk, ${ }^{2}$ Catalin Miron, ${ }^{3}$ Kiyoshi Ueda, ${ }^{4}$ Piero Decleva, ${ }^{5}$ and Fernando Martín ${ }^{1,6, *}$ \\ ${ }^{1}$ Departamento de Química, Módulo 13, Universidad Autónoma de Madrid, ES-28049 Madrid, Spain \\ ${ }^{2}$ Department of Physics and Astronomy, University of Turku, FI-20014 Turku, Finland \\ ${ }^{3}$ Synchrotron SOLEIL, L'Orme des Merisiers, Saint-Aubin, BP 48, FR-91192 Gif-sur-Yvette Cedex, France \\ ${ }^{4}$ Institute of Multidisciplinary Research for Advanced Materials, Tohoku University, Sendai 980-8577, Japan \\ ${ }^{5}$ Dipartimento di Scienze Chimiche, Universita' di Trieste, IT-34127 Trieste, and CNR-IOM, Trieste, Italy \\ ${ }^{6}$ Instituto Madrileño de Estudios Avanzados en Nanociencia (IMDEA-Nanociencia), Cantoblanco, ES-28049 Madrid, Spain
}

(Received 16 September 2011; revised manuscript received 17 January 2012; published 16 February 2012)

\begin{abstract}
Current techniques based on $\mathrm{x}$-ray or electron diffraction are successfully employed for structure determination in condensed matter but are sometimes limited when applied to low density media such as the gas phase. Here we show that vibrationally resolved photoelectron spectroscopy based on $\mathrm{x}$ rays generated by third generation synchrotron light sources can be used to infer the structure of isolated molecules in a simple and efficient way. In particular, we show that vibrational ratios obtained from inner shell $\mathrm{C} 1 \mathrm{~s}$ photoelectron spectroscopy of isolated methane molecules exhibit pronounced oscillations that are the fingerprints of electron diffraction by the surrounding atomic centers, thus providing the necessary information for the determination of the molecular geometry.
\end{abstract}

DOI: 10.1103/PhysRevA.85.023409

PACS number(s): 33.80.Eh, 34.10.+x, 34.50.-s

\section{INTRODUCTION}

Probing with diffraction the structure of matter at the atomic scale requires the use of light or particle beams with wavelengths of the order of the characteristic interatomic distances. Due to their short wavelength, $\mathrm{x}$ rays and fewhundred-electron-volts electron beams are thus the ideal sources. For example, they can efficiently diffract at crystals, exactly as normal waves do when they strike a diffraction grating. From the observed diffraction patterns, one can then deduce quantitative structural information such as lattice constants and symmetry [1-4]. When used with ultrahigh time resolution, they even allow one to map structural changes as those occurring in phase transitions [5,6]. $\mathrm{X}$ rays can also efficiently ionize the inner shells of the atomic or molecular constituents, thus producing electrons that scatter coherently (i.e., diffract) within the surroundings of the ionized atom or molecule. The NEXAFS (near-edge x-ray absorption fine structure) or EXAFS (extended x-ray absorption fine structure) techniques are based on this principle [7,8]. The fingerprints of this coherent electron scattering in the measured spectra provides valuable information about the local electronic structure and coordination geometries $[9,10]$. Thus, photoelectron diffraction is used extensively in solid state [11] and surface physics $[12,13]$ to obtain structural information in crystals as well as in bulk amorphous materials

When these techniques are applied to gases at low pressure to determine, for example, the structure of an isolated molecule, one has to face a severe disadvantage: the extremely low density of the medium in comparison with that of bulk materials, which, in some cases, prevents one from observing any diffraction at all. Diffraction is clearly observed in the condensed phase because the relatively low number of photoelectrons generated by standard x-ray sources is amply compensated by the large number of atoms present in the solid

\footnotetext{
*fernando.martin@uam.es
}

or liquid sample (which is of the order of Avogadro's number, i.e., $\left.\sim 10^{23}\right)$. In the case of low density media, a possible solution is to increase the intensity of the light source, so that either more $\mathrm{x}$ rays are efficiently diffracted or the outgoing flux of diffracted photoelectrons is larger. Thus, high intensity, $\mathrm{x}$-ray free electron lasers (XFEL) have been recently proposed as the ideal tool for measurements on isolated species $[14,15]$. For example, they have already provided evidence of $\mathrm{x}$-ray diffraction by single nanocrystals of large membrane protein complexes [16] and by a single virus particle [17]. Photoelectrons arising from XFEL irradiation of low-pressure gases have also been suggested as a way to perform time-resolved imaging of molecular structure (i.e., the "molecular movie") by using so-called photoelectron holography [18], which in some way reminds us the EXAFS spectroscopy. This success is limited, however, by the complexity, large size (up to 4-5 km) and high cost of XFEL single-user facilities. For this reason, it is worth exploring more handy alternatives, as those provided by third-generation synchrotron radiation sources in combination with high kinetic energy resolution detection techniques. As shown in this paper, we propose that vibrationally resolved photoelectron spectroscopy measurements performed with such synchrotron radiation sources [19] is a useful alternative.

Our proposal is inspired by recent work on diatomic molecules. First, by valence-shell photoionization of $\mathrm{H}_{2}, \mathrm{~N}_{2}$, and $\mathrm{CO}$, in which the ratios between vibrationally resolved photoionization cross sections (hereafter called $v$ ratios) oscillate with photon energy [20,21]. The oscillations are due to the coherent electron emission from the two atomic centers and their frequency is dictated by the value of the internuclear distance, similarly to what happens in a classical Young's double-slit experiment. The experiments have thus been able to provide the information needed to characterize the geometry of a diatomic molecule: the bond length. Second, by the fact that in all molecules inner-shell molecular orbitals are localized on the atomic centers, so that electrons can be effectively ejected from a very localized region of space 
and can be subsequently scattered by the neighboring atomic centers thus leading to diffraction. $K$-shell photoionization experiments on isolated fixed-in-space $\mathrm{CO}$ molecules have shown evidence of such scattering processes in photoelectron angular distributions [22,23]. Third, by the recently observed diffraction of laser-induced tunneling electrons produced by intense infrared femtosecond pulses on $\mathrm{N}_{2}$ and $\mathrm{O}_{2}$ [24,25]. In these experiments the oscillating electric field of the laser forces a fraction of the tunneled electron to go back to the parent ion where it can diffract.

The specific example we consider here is vibrationally resolved $\mathrm{C}(1 s)$ photoionization of $\mathrm{CH}_{4}$, a molecule for which there is a vast literature [26-29], but no evidence of diffraction has been reported so far. This is partly due to the need for high kinetic-energy resolution in combination with high photon energy (that of $\mathrm{x}$ rays) but also to the fact that emission of a fast electron is accompanied by recoil of the residual cation [28], thus leading to a redistribution of the available energy even on symmetry-forbidden vibrational modes. As shown by Kukk et al. [28], the recoil of $\mathrm{CH}_{4}^{+}$ions leads to excitation of the asymmetric stretching and bending vibrations, which superimpose to the dominant symmetric stretching vibrational components. As a result, the apparent $v$ ratios increase almost linearly with photon energy, thus hiding the underlying diffraction. In this work we present results from (i) a theoretical approach that describes both the electronic and nuclear degrees of freedom, (ii) experiments performed at the SOLEIL synchrotron (France) that improve by about a factor of 5 statistical errors reported in previous experiments at the SPring- 8 synchrotron, and (iii) a data analysis of these new data and of previously reported SPring- 8 measurements that allow us to separate the diffraction contribution from the recoil contribution. As we will show below, the calculated and measured $v$ ratios so obtained exhibit pronounced oscillations as a function of photoelectron energy that are unambiguously due to scattering of the ejected electron by the surrounding hydrogen atoms. A fit of these $v$ ratios to a simple model formula thus allows one to determine the molecular geometry.

\section{FIRST-PRINCIPLES CALCULATIONS}

The vibrationally resolved photoionization cross section is evaluated to first order of perturbation theory within the Born-Oppenheimer and dipole approximations:

$$
\sigma_{\alpha}\left(v, v^{\prime}, \omega\right)=\frac{4 \pi^{2} \omega}{3 \hbar c} a_{0}^{2} \sum_{\eta} \sum_{l_{\eta}}\left|T_{\alpha_{\eta} l_{\eta} v v^{\prime}}(\varepsilon)\right|^{2},
$$

where

$$
T_{\alpha_{\eta} l_{\eta} v v^{\prime}}(\varepsilon)=\int \chi_{M, v}^{*}(R) \mu_{\alpha_{\eta} l_{\eta}}(\varepsilon, R) \chi_{M_{\alpha}^{+}, v^{\prime}}(R) d R,
$$

$\mu_{\alpha_{\eta} l_{\eta}}$ is the dipole-transition matrix element between the initial electronic state $\psi_{0}$ and the final electronic continuum state $\psi_{\alpha_{\eta} l_{\eta}}$ of $\mathrm{CH}_{4}, \alpha$ denotes the electronic state of the residual ion, $\eta$ is the symmetry of the final state, $\chi_{M, v}$ is the initial vibrational state, $\chi_{M_{\alpha}^{+}, v^{\prime}}$ is the final vibrational state, $\omega$ is the photon energy, and $\varepsilon$ is the photoelectron energy.

To evaluate the electronic continuum state $\psi_{\alpha_{\eta} l_{\eta}}$, we have used an extension of density functional theory (DFT) originally developed by Decleva and co-workers to treat molecular ionization at the molecule's equilibrium position [30]. The method has been shown to provide accurate photoionization cross sections for simple as well as for very complex molecules (see, e.g., Ref. [30]). Here the method has been extended to describe nonequilibrium geometries. In particular, we have considered 60 different geometries associated with the symmetric stretching mode. They have been built by varying synchronously the four C-H distances. A standard local density approximation (LDA) functional has been used to describe exchange and correlation effects.

For each value of the $\mathrm{C}-\mathrm{H}$ distance, we have followed the following procedure. The initial Kohn-Sham orbitals have been generated by running a standard preliminary LCAO-DFT calculation with the program Amsterdam density functional (ADF) using a LDA functional in a double $\zeta$-polarization (DZP) basis set. The resulting sets of Slater type orbitals are then used to build the Hamiltonian matrix in a new basis set of $B$-spline functions $B$ and real spherical harmonics $Y^{R}$ :

$$
\xi_{\alpha l_{\alpha} j}^{i}(r, \theta, \phi)=\sum_{k=1}^{N_{\mathrm{eq}}^{i}} \frac{1}{r_{k}} B_{j}\left(r_{k}\right) \sum_{m_{\alpha}=-l_{\alpha}}^{l_{\alpha}} b_{\alpha l_{\alpha} m} Y_{l_{\alpha} m}^{R}\left(\theta_{k}, \phi_{k}\right)
$$

where $\alpha$ gathers the indexes referring to a specific irreducible representation (IR), $l_{\alpha}$ and $m_{\alpha}$ correspond to the usual angular momentum quantum numbers, $i$ and $k$ indicate, respectively, the $i$ th nonequivalent expansion center and its $k$ th equivalent center, $N_{\text {eq }}^{i}$ is the number of equivalent centers associated to $i$, the index $j$ refers to the $j$ th $B$ spline, $r, \theta$, and $\phi$ stand for the spherical coordinates in the molecular framework, and $b_{\alpha l_{\alpha} m}$ are the coefficients of the symmetry adapted linear combination of real spherical harmonics. The nonequivalent centers are divided into two categories:

(1) The one-center expansion (OCE), which is unique (as its name implies) and located on a chosen origin. Its radius is large (40 a.u. in the present calculation) which allows us to correctly treat the long range oscillatory behavior of the continuum wave function.

(2) The off-centers, which are located on each nonequivalent nuclei in order to describe more accurately the sharpness of the bound state wave functions. The expansion interval is cut off and is generally quite small (0.5 a.u. in the present calculation) in order to avoid linear dependencies due to excessive overlap with other basis functions. It has been shown that addition of the off-centers to the OCE $B$-spline basis improves dramatically the convergence of the calculation for most molecules.

In the particular case of methane, the OCE is centered on the carbon atom and only one nonequivalent off-center is required to take care of the hydrogen atoms. Then the radial part of the basis functions is defined by two sets of $N_{b}$ onedimensional $B$-spline functions, built on two different radial interval: $\left[0 ; R_{\max }^{0}\right]$ for the set $\left\{\xi_{\alpha l_{\alpha}}^{0}\right\}$ and $\left[0 ; R_{\max }^{i}\right]$ for the set $\left\{\xi_{\alpha l_{\alpha}}^{i}\right\}$. The LCAO basis set and consequently the cost and accuracy of the calculation is completely defined by

(1) The $B$-spline order and the $R_{\max }^{0}, R_{\max }^{i}$, and $N_{b}$ parameters.

(2) The maximum value of the angular momentum expansion $L_{\max }^{0}$ for the OCE and $L_{\max }^{i}$ for the off-center sets. 
Since the off-center radius spheres cannot intersect each other, the resulting Hamiltonian matrix $\mathbf{H}$ is partitioned into submatrices which are the diagonal elements $\mathbf{H}_{j j}$ and the blocks $\mathbf{H}_{0 j}$ and $\mathbf{H}_{j 0}$ between the set $\left\{\xi_{\alpha l_{\alpha}}^{0}\right\}$ and $\left\{\xi_{\alpha l_{\alpha}}^{i}\right\}$. The calculation of the nondiagonal block elements $\mathbf{H}_{0 j}$ and $\mathbf{H}_{i j}$ represents the main computational effort because of the lack of analytical resolution. To carry out the integration, a numerical three-dimensional Gauss-Legendre scheme is employed. Also, a rotation of the coordinate framework of the spherical harmonics of the main center along the polar axis associated with the subsystems defined by the expansion centers leads to significant improvement. This exploits local cylindrical symmetry reducing integration to two variables $(r$ and $\varphi)$.

The bound states are extracted by a generalized diagonalization of $\hat{H}_{\mathrm{KS}}$ and continuum states by block inverse iteration [31] for each given photoelectron energy. Electronic dipole matrix elements were computed for 200 photoelectron energies, in a basis of $400 \mathrm{~B}$ splines, a box size of 40 a.u. and a maximum $L$ value of 13. Making use of the accurate Morse potential parameters given in Ref. [32] $\left(\mathrm{CH}_{4}: \omega_{e}=\right.$ $3059.9 \mathrm{~cm}^{-1}, \omega_{e} x_{e}=13.7 \mathrm{~cm}^{-1} ; \mathrm{CH}_{4}^{+1 s}: \omega_{e}=3383.5 \mathrm{~cm}^{-1}$ and $\omega_{e} x_{e}=15.8 \mathrm{~cm}^{-1}$ ), we have evaluated the vibrational wave functions by diagonalizing the vibrational hamiltonian in a basis of $1000 \mathrm{~B}$ splines within a box of 10 a.u.

\section{EXPERIMENTAL METHOD}

The new measurements have been carried out at the PLEIADES beamline (10-1000 eV) dedicated to ultrahigh resolution spectroscopic studies of isolated species [33-36] at the $2.75 \mathrm{GeV}$ French national synchrotron radiation laboratory SOLEIL. The quasiperiodic Apple II elliptical undulator of $80 \mathrm{~mm}$ period (HU80) has been used to generate arbitrarily tilted linearly polarized light. In the experiments, a 1600 lines/mm grating has been used, while the monochromator slit has been adjusted to keep the spectral broadening fixed at $50 \mathrm{meV}$. Photoelectron spectra have been collected at the magic angle of $57.4^{\circ}$ with respect to the light polarization vector using a wide angle lens VG Scienta R4000 electron spectrometer operated at $50 \mathrm{eV}$ pass energy and mounted with the electron detection axis lying vertical in the plane perpendicular to the x-ray beam propagation axis. Pure methane (99.9\%) from Air Liquide has been introduced using a differentially pumped gas cell allowing tunable compensation of the plasma potentials associated with the electric field created in the interaction region by the ion density gradient along the photon beam. The electron spectrometer resolution was set to $60 \mathrm{meV}$. Additional causes such as translational (20-45 meV) and rotational Doppler broadening $[37,38]$ lead to a total Gaussian broadening of SOLEIL spectra of $85 \mathrm{meV}$ at $330 \mathrm{eV}$ photon energy.

Vibrational progressions in the $\mathrm{C} 1 s$ photoelectron spectra were analyzed using the least-squares curve fitting macro package SPANCF [39] for Igor Pro. All spectra from each series of measurements were analyzed as a set with linked parameters. The progressions were decomposed into the symmetric stretch, asymmetric bending, and asymmetric stretch modes. The first is excited due to the molecular potential change upon core ionization and the last two are excited by the photoelectron recoil momentum delivered to the central carbon atom. The vibrational frequencies of the recoil-excited bending mode and asymmetric stretching mode were fixed to 179 and $414 \mathrm{meV}$, respectively [32], and the peak intensities were calculated according to the recoil model [40]. These two progressions thus formed a background for the symmetric stretch progression to be fitted. The peak separations were free fitting parameters for this progression, but they were linked to be the same within the set of spectra. The values of 397.0, 396.4, and $395.9 \mathrm{meV}$ were obtained from the highest quality SOLEIL data set for the $v=(1-0),(2-1)$, and (3-2) separations, respectively. Post-collision-interaction (PCI) modified Voigt profiles [41] were used for fitting with the PCI asymmetry calculated from the photon and average Auger electron energies, neglecting angular anisotropy effects. Common Lorentzian lifetime width of $99 \mathrm{meV}$ was obtained for the SOLEIL data set, but for other, SPring8 data sets, the Lorentzian components of 92 and $97 \mathrm{meV}$ gave the best fit. The slight discrepancy could be due to the deviation of the instrument function from pure Gaussian shape, depending on the experimental setup used. These values nevertheless bracket closely the earlier reported lifetime width of $95 \pm 5 \mathrm{meV}$ [26].

The peak intensity ratios of the symmetric stretch progression is the main result of the fitting procedure. These ratios $R_{m}$ have been calculated as

$$
R_{m}=\frac{R_{h}+2 c R_{v}}{1+2 c}
$$

where $R_{h}$ and $R_{v}$ are the measured peak intensity ratios along and perpendicular to the polarization, respectively, and

$$
c=\frac{1-\beta / 2}{1+\beta} .
$$

The anisotropy parameters $\beta$ have been taken from Ref. [42].

We have used the same fitting procedure to reanalyze previous measurements performed at SPring8 (Japan). For photon energies at and above $340 \mathrm{eV}, \beta$ is close to 2 , so that $c \rightarrow 0$ and only $R_{h}$ is shown. The error bars of the ratios are from the statistical uncertainty of the peak areas and, for the error bars of the calculated $R_{m}$ values, the uncertainty of $\beta$ was assumed \pm 0.05 .

\section{RESULTS}

Figure 1(b) shows the photoelectron spectra for the symmetric stretching mode at a photon energy of $330 \mathrm{eV}$. The experimental data have been renormalized to the theoretical results, which are shown by vertical bars. As can be seen, $\mathrm{CH}_{4}$ photoionization mainly leads to $\mathrm{CH}_{4}^{+}$ions in the ground vibrational state $v=0$ of its stretching mode. This is because the equilibrium geometries of $\mathrm{CH}_{4}$ and $\mathrm{CH}_{4}^{+}$are very similar. Indeed, as illustrated in Fig. 1(a), a vertical one-photon transition from the ground state of $\mathrm{CH}_{4}$ necessarily leads to $\mathrm{CH}_{4}^{+}$ions with a geometry close to that of the minimum of the potential energy surface (PES), so that the maximum Franck-Condon overlap corresponds to the $v=0 \rightarrow v^{\prime}=0$ transition. Consequently, the intensity of the observed peaks decreases rapidly with the degree of vibrational excitation of the remaining $\mathrm{CH}_{4}^{+}$ion and, for all practical purposes, it is negligible beyond $v^{\prime}=2$. The intensity of the measured peaks decreases very rapidly with photon energy. This rapid decrease, which also occurs in atoms, complicates the identification of 

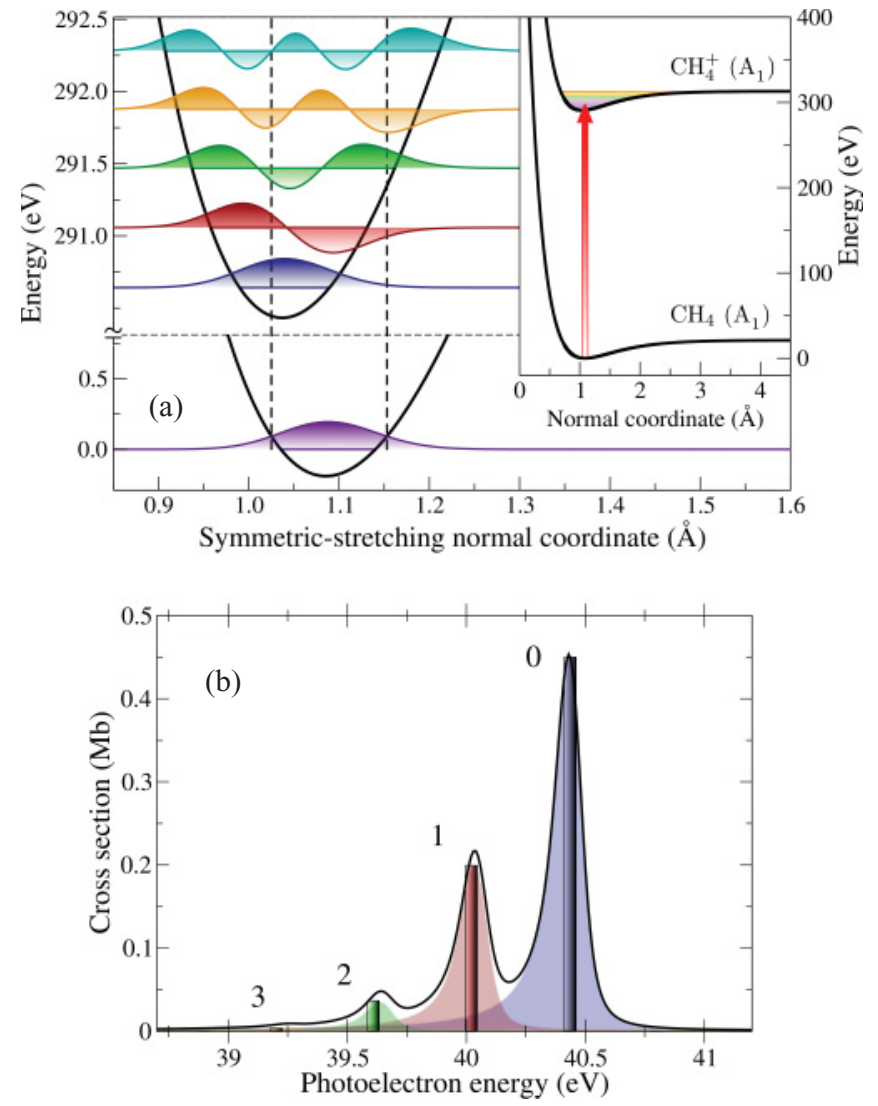

FIG. 1. (Color online) (a) Potential energy curves of the ground states of $\mathrm{CH}_{4}\left(A_{1}\right)$ and $\mathrm{CH}_{4}^{+}\left(A_{1}\right)$ along the symmetric-stretching normal coordinate. The vibrational wave functions associated with the relevant vibrational states are also shown. The vertical red arrow indicates a vertical one-photon transition. The vertical dashed lines enclose the Franck-Condon region. (b) Photoelectron spectra of methane taken at $h v=330 \mathrm{eV}$. Experimental results: full curve. Theoretical results: bars. Peak labels indicate the vibrational quantum number $v^{\prime}$ of $\mathrm{CH}_{4}^{+}$.

intrinsic molecular effects in the photoelectron spectrum. A very elegant way of removing this rapid decrease from the analysis is to consider $v$ ratios, in which such a behavior is no longer present because both the numerator and the denominator decrease with a similar rate.

Figure 2 shows the measured and calculated $v$ ratios as functions of photon energy. As can be seen, the $v$ ratios exhibit pronounced oscillations superimposed to a nearly flat background. The agreement between theory and the results of the SOLEIL experiment is excellent, even for the very small $v=2 / v=0$ ratio (notice that experimental statistical errors are significantly smaller than in previously reported measurements). The oscillations are also visible, although with larger error bars, in the experiment performed a few years ago at the SPring8 synchrotron (Japan) after removing from the $v$ ratios the contribution of the asymmetric modes due to recoil (data shown in Fig. 2).

\section{SCATTERING MODEL}

To provide more physical insight into these results, we have performed model one-electron calculations in which the initial electronic state of $\mathrm{CH}_{4}$ is represented by the $1 s$ orbital

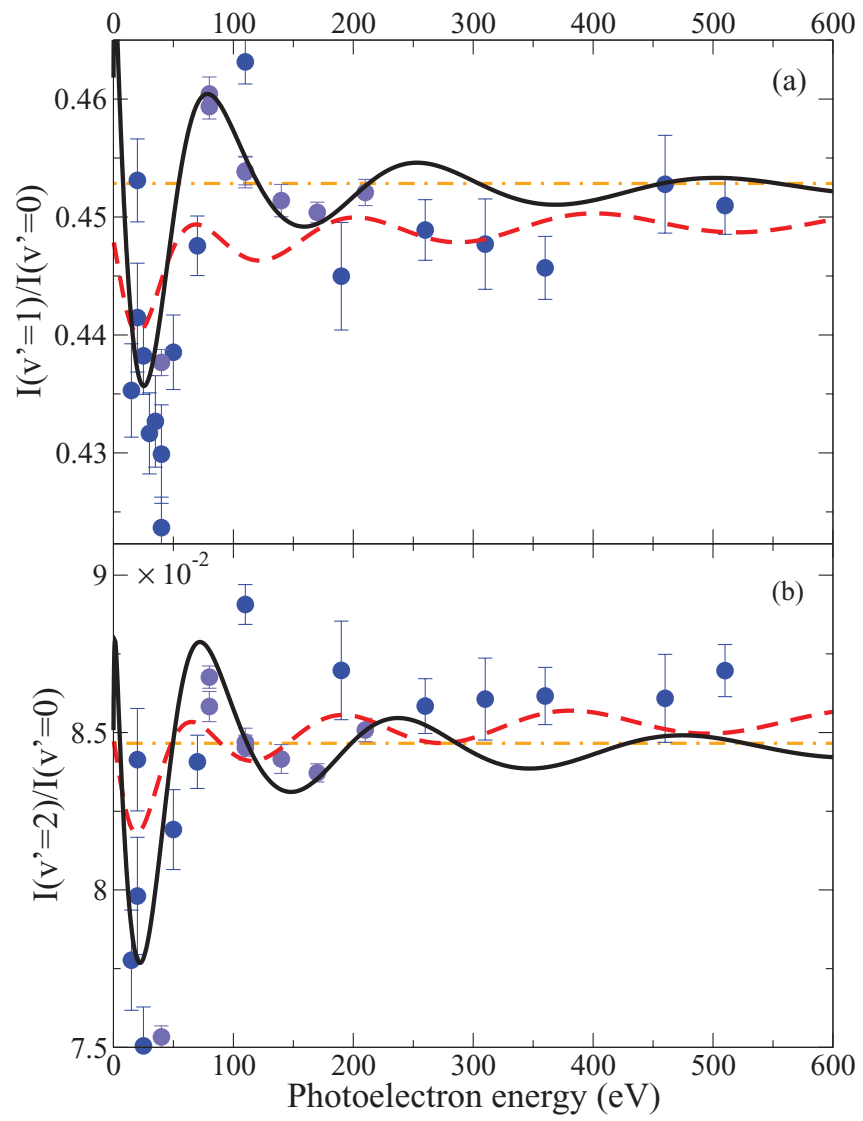

FIG. 2. (Color online) Ratios between vibrationally resolved photoionization cross sections in $\mathrm{C} 1 \mathrm{~s}$ photoionization of $\mathrm{CH}_{4}$ for the $\mathrm{CH}_{4}^{+}$symmetric stretching mode. (a) $v^{\prime}=1 / v^{\prime}=0$ ratio. (b) $v^{\prime}=2 / v^{\prime}=0$ ratio. Blue (dark gray) circles: experimental data of Ref. [28] obtained at SPring8 after removing the recoil contributions. Violet (light gray) circles: present experimental data. Both data sets include the statistical and systematic errors. Black full lines: present theoretical results. Red dashed lines: results of the simple model explained in the text. Horizontal dashed-dotted lines: ratios predicted by the Franck-Condon approximation. The photoelectron energy is given by $\left[h v-V_{\text {ion }}(\mathrm{C} 1 s)\right]$, where $V_{\text {ion }}(\mathrm{C} 1 s)=290.6 \mathrm{eV}$ is the energy required to remove an electron from the $\mathrm{C} 1 \mathrm{~s}$ orbital of $\mathrm{CH}_{4}$.

of the $\mathrm{C}$ atom, $\phi_{1 s, \mathrm{C}}$, and the final continuum state of the ionized electron by a plane wave plus a first order correction representing the scattering from the central $\mathrm{C}$ atom and the four $\mathrm{H}$ atoms, $\psi_{\vec{k}}^{-}(\vec{r})=\exp (i \vec{k} \cdot \vec{r})+\psi_{\mathrm{C}, \vec{k}}^{-}(\vec{r})+\psi_{\mathrm{H}, \vec{k}}^{-}(\vec{r})$. Within this model the transition dipole matrix elements have been evaluated as functions of the symmetric stretching normal coordinate and then used to obtain vibrationally resolved photoionization cross sections.

In more detail, the vibrationally resolved photoionization cross section $\sigma_{v}$ from the carbon $1 s$ core orbital $\phi_{1 s, \mathrm{C}}$ of methane in the ground vibrational state is estimated within the single-active-electron approximation

$$
\sigma_{v}=2 \frac{4 \pi^{2} k}{c \omega} \int d \Omega_{k}\left|\left\langle\psi_{\vec{k}}^{-} \chi_{v}^{\text {ion }}|\hat{\epsilon} \cdot \vec{p}| \phi_{1 s, \mathrm{C}} \chi_{\mathbf{0}}\right\rangle\right|^{2}
$$

In the final state, the $\mathrm{CH}_{4}^{+}$parent ion in the (possibly excited) vibrational state $\chi_{v}^{\text {ion }}$ is coupled to an electron state in the 
continuum $\psi_{\vec{k}}^{-}$with asymptotic momentum $\vec{k}$. The latter is computed at the level of the first-order Born approximation,

$$
\left|\psi_{\vec{k}}^{-}\right\rangle=|\vec{k}\rangle+G_{0}^{-} V|\vec{k}\rangle, \quad V=V_{\mathrm{C}}+\sum_{i=1}^{4} V_{\mathrm{H}_{i}},
$$

where the interaction between the photoelectron and the parent ion is represented with an effective potential given by a sum of screened atomic charges

$$
V_{\mathrm{C}}=\frac{1-\left(Z_{1 s, \mathrm{C}}^{*}-1\right) e^{-\left|\vec{r}-\vec{R}_{\mathrm{C}}\right|}}{\left|\vec{r}-\vec{R}_{\mathrm{C}}\right|}, \quad V_{\mathrm{H}_{i}}=\frac{e^{-\left|\vec{r}-\vec{R}_{\mathrm{H}_{i}}\right|}}{\left|\vec{r}-\vec{R}_{\mathrm{H}_{i}}\right|} .
$$

According to Slater's prescription, the nuclear effective charge felt by the electron in the $\mathrm{C}(1 s)$ orbital is $Z_{1 s, \mathrm{C}}^{*}=5.7$. At large distances, $V$ reduces to the Coulomb potential of the parent ion's single residual charge. In momentum coordinates, the expression for the electronic part of the final wave function reads

$$
\psi_{\vec{k}}^{-}(\vec{p}) \simeq \delta(\vec{p}-\vec{k})+\psi_{\mathrm{C}, \vec{k}}^{-}(\vec{p})+\psi_{\mathrm{H}, \vec{k}}^{-}(\vec{p}),
$$

where

$$
\begin{aligned}
& \psi_{\mathrm{C}, \vec{k}}^{-}(\vec{p})=-\frac{1}{\pi^{2}} \frac{e^{-i(\vec{p}-\vec{k}) \cdot \vec{R}_{\mathrm{C}}}}{(k+p)\left(k-p-i 0^{+}\right)} \\
& \times\left(\frac{1}{|\vec{p}-\vec{k}|^{2}}+\frac{Z_{1 s, \mathrm{C}}^{*}-1}{|\vec{p}-\vec{k}|^{2}+\lambda_{\mathrm{C}}^{2}}\right), \\
& \psi_{\mathrm{H}, \vec{k}}^{-}(\vec{p})=-\frac{1}{\pi^{2}} \frac{1}{(k+p)\left(k-p-i 0^{+}\right)} \sum_{i} \frac{e^{-i(\vec{p}-\vec{k}) \cdot \vec{R}_{\mathrm{H}_{i}}}}{|\vec{p}-\vec{k}|^{2}+\lambda_{\mathrm{H}}^{2}} .
\end{aligned}
$$

In principle, the factors $e^{-i \Delta \vec{k} \cdot \vec{R}}$ in the scattering components $\psi_{\mathrm{C}, \vec{k}}^{-}$and $\psi_{\mathrm{H}, \vec{k}}^{-}$couple the photoelectron to all the degrees of freedom of the nuclei. In practice, the dominant monopolar term $\sin (\Delta k R) / \Delta k R$ affects just the totally symmetric stretching mode; hence, we will focus on the excitation of the latter, assuming $v=0$ for all the other vibrational modes. When computing the square module of the transition amplitude, Coulomb corrections are retained to first order only

$$
\begin{aligned}
\mid\left\langle\psi_{\vec{k}}^{-}\right. & \left.\chi_{\boldsymbol{v}}^{\text {ion }}|\hat{\epsilon} \cdot \vec{p}| \phi_{1 s, \mathrm{C}} \chi_{\mathbf{0}}\right\rangle\left.\right|^{2} \\
\simeq & \left|\left\langle\chi_{\boldsymbol{v}}^{\text {ion }} \mid \chi_{\mathbf{0}}\right\rangle\right|^{2} \phi_{1 s, \mathrm{C}}^{2}(k)(\vec{k} \cdot \hat{\epsilon})^{2}+2 \phi_{1 s, \mathrm{C}}(k)(\vec{k} \cdot \hat{\epsilon}) \\
& \times\left\langle\chi_{\boldsymbol{v}}^{\text {ion }} \mid \chi_{\mathbf{0}}\right\rangle\left\langle\chi_{\mathbf{0}}\right| \operatorname{Re}\left(\left\langle\phi_{1 s, \mathrm{C}}|\hat{\epsilon} \cdot \vec{p}| \psi_{\mathrm{H}, \vec{k}}^{-}\right\rangle\right. \\
& \left.+\left\langle\phi_{1 s, \mathrm{C}}|\hat{\epsilon} \cdot \vec{p}| \psi_{\mathrm{C}, \vec{k}}^{-}\right\rangle\right)\left|\chi_{\boldsymbol{v}}^{\text {ion }}\right\rangle .
\end{aligned}
$$

In this picture the violation of the Franck-Condon principle is caused by the scattering of the photoelectron by the hydrogen atoms incorporated in the second term on the right-hand side of (10).

The total cross section is thus given by

$$
\begin{aligned}
\sigma_{v} \simeq & \frac{32 \pi^{3} k}{3 c \omega} \phi_{1 s, \mathrm{C}}^{2}(k)\left|\left\langle\chi_{\boldsymbol{v}}^{\text {ion }} \mid \chi_{\mathbf{0}}\right\rangle\right|^{2} \\
& +\frac{16 \pi^{2} k}{c \omega} \phi_{1 s, \mathrm{C}}(k)\left\langle\chi_{\boldsymbol{v}}^{\text {ion }} \mid \chi_{\mathbf{0}}\right\rangle\left\langle\chi_{\mathbf{0}}|F(k ; \lambda, \boldsymbol{R})| \chi_{\boldsymbol{v}}^{\text {ion }}\right\rangle .
\end{aligned}
$$

The function $F(k ; \lambda, \boldsymbol{R})$ accounts for the scattering of the photoelectron by the hydrogen atoms, which leads to oscillations in the photoionization cross section, as well as for the non-Franck-Condon character of the excitation of the symmetric stretching mode. $F(k ; \lambda, \boldsymbol{R})$ has the following expression:

$$
\begin{aligned}
F(k ; \lambda, \boldsymbol{R})= & -\frac{8}{3} \sum_{i} Z_{i} \int p^{2} d p \phi_{1 s, \mathrm{C}}(p) \mathrm{P} \frac{1}{k^{2}-p^{2}} \\
& \times \int_{-1}^{1} d \cos \theta \frac{p k \cos \theta}{|\vec{p}-\vec{k}|^{2}+\lambda_{i}^{2}} \frac{\sin |\vec{p}-\vec{k}| d_{\mathrm{CA}_{i}}}{|\vec{p}-\vec{k}| d_{\mathrm{CA}_{i}}},
\end{aligned}
$$

where $d_{\mathrm{CA}_{i}}$ is the distance between the central carbon atom and the $i$ th atom in the effective potential. Notice that, within the present approximations, the two terms corresponding to the carbon atom do not give rise to any vibronic coupling. The angular integral has a closed solution in terms of the exponential integral $E_{1}(z)$, a well known special function [43]. The integral over the momentum $p$, as well as that between symmetric vibrational wave functions must instead be carried out numerically.

The above formulas have been evaluated by using the same vibrational wave functions as in the $a b$ initio calculations described in Sec. II. The results of the model are shown by dashed lines in Fig. 2. For photoelectron energies larger than $50-100 \mathrm{eV}$, where the first-order correction to the plane wave description of the continuum electron is a reasonable approximation, the model reproduces quite nicely the amplitude and approximate periodicity $\pi / R_{\mathrm{CH}}$ (where $R_{\mathrm{CH}}$ is the $\mathrm{C}-\mathrm{H}$ distance at the equilibrium geometry) of the oscillations. The phase is not well described because electron delocalization and correlation effects, not included in the model, are responsible for additional phase shifts in the scattering wave function. The relative importance of the different terms contributing to the continuum wave function $\psi_{\vec{k}}^{-}(\vec{r})$ is illustrated in Fig. 3. This figure shows that neglect of the $\psi_{\mathrm{C}, \vec{k}}^{-}(\vec{r})$ term, that is, of the scattering from the central $\mathrm{C}$ atom, has a negligible effect in

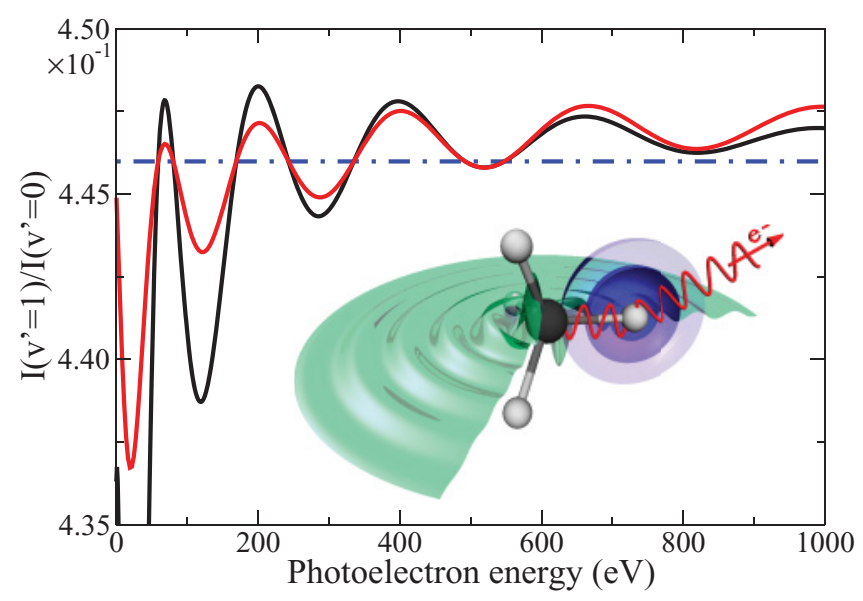

FIG. 3. (Color online) Origin of the oscillations in the $v$ ratios. Black line: results from the full model described in the text. Red (light gray) line: results obtained by excluding scattering of the ejected electron by the central $\mathrm{C}$ atom. Blue dashed-dotted line: results obtained by excluding scattering of the ejected electron by the peripheral $\mathrm{H}$ atoms. The latter curve simply corresponds to the ratio between the Franck-Condon factors for the two final vibrational states of the $\mathrm{CH}_{4}^{+}$ion. 
the $v$ ratios beyond $300 \mathrm{eV}$. In contrast, neglect of the $\psi_{\mathrm{H}, \vec{k}}^{-}(\vec{r})$ term, that is, of the scattering by the four $\mathrm{H}$ atoms, leads to no oscillations at all. Therefore the amplitude and frequency of the oscillations is entirely explained by scattering from the surrounding $\mathrm{H}$ atoms (as illustrated by the inset in Fig. 3). Scattering from the $\mathrm{C}$ atom plays some role below $300 \mathrm{eV}$ by decreasing the amplitude of the oscillations due to scattering by the $\mathrm{H}$ atoms.

In order to fit the measured $v$ ratios, it is much more useful to use a further simplification of the above expression [44], which leads to the following analytical formula:

$$
\begin{aligned}
\frac{\sigma_{1}}{\sigma_{0}} \simeq & A+B e^{-k^{2} / 26}\left[0.42 \cos \left(2 k \bar{R}_{\mathrm{CH}}+\phi\right)\right. \\
& \left.-\sin \left(2 k \bar{R}_{\mathrm{CH}}+\phi\right) / k\right] .
\end{aligned}
$$

This expression contains four free parameters: $A$ is an adjusted Franck-Condon ratio, $B$ controls the oscillation amplitude, $\bar{R}_{\mathrm{CH}}$ is the average $\mathrm{C}-\mathrm{H}$ distance, and $\phi$ is a phase shift. Although, strictly speaking, $\phi$ is a function of photoelectron energy, we have assumed that it is a constant since the largest variation with photoelectron energy comes from the $2 k \bar{R}_{\mathrm{CH}}$ term. We have fitted the experimental data in the range $40-600 \mathrm{eV}$ (in this way we exclude the low-energy region in which electron correlation is important). The result of the fit is $\bar{R}_{0}=1.1 \pm 0.1 \AA, A=0.452 \pm 0.01, B=0.020 \pm 0.004$, and $\phi=(0.5 \pm 0.4) \pi$. The value of the $\mathrm{C}-\mathrm{H}$ bond distance is in good agreement with that obtained from spectroscopic data [45].

It is worth noticing here that, as shown in Ref. [20] for the case of coherent two-center photoelectron emission from diatomic molecules, the oscillatory structures observed in the $v$ ratios carry structural information associated with both the initial neutral molecule and the final molecular cation. This is because the photoelectron probes regions of internuclear distances that are different for each final vibrational state of the cation. The geometries of $\mathrm{CH}_{4}$ and $\mathrm{CH}_{4}^{+}$are so similar that the model used here assumes that both species have identical geometries. However, disentangling the structure of the neutral molecule and the resulting molecular cation when their geometries are significantly different requires a more elaborate treatment along the lines described in Ref. [20].

\section{CONCLUSION}

We have shown that the observation of pronounced oscillations in the $\mathrm{CH}_{4} v$ ratios as a function of photoelectron energy is unambiguously due to diffraction of the ejected electron by the surrounding $\mathrm{H}$ atoms. The imprint of the molecular geometry can be recovered from the experimental data by using a simple model. Thus, in a more general context, the observation of oscillatory patterns in the $v$ ratios can offer a handle to the determination of molecular geometry.

\section{ACKNOWLEDGMENTS}

We thank Mare Nostrum BSC, Cineca, and CCC-UAM for allocation of computer time. Work supported by the MICINN Project Nos. FIS2010-15127, ACI2008-0777, and CSD 200700010 (Spain), the European COST Action CM0702 and the Marie Curie ITN CORINF (EU), the XCHEM Advanced Grant 290853 of the European Research Council, the MEXT and JSPS agencies (Japan), and the Academy of Finland. Data collection was performed on the PLEIADES beamline at SOLEIL Synchrotron, France. We are grateful to T. D. Thomas for stimulating discussions, to the SOLEIL and SPring-8 staff for smoothly running the facilities, and to C. Nicolas, M. Patanen, O. Travnikova, U. Hergenhahn, X.-J. Liu, G. Prümper, H. Yoshida, C. Makochekanwa, T. Tanaka, M. Kitajima, H. Tanaka, A. Pavlychev, T. Sunami, F. Tahara, A. De Fanis, and Y. Tamenori, for their contribution in the collection of experimental data.
[1] G. H. Stout and L. H. Jensen, X-Ray Structure Determination, A Practical Guide, 2nd ed. (Wiley, New York, 1989).

[2] D. L. Dorset, Structural Electron Crystallography (Plenum, New York, 1995).

[3] C. Baerlocher, F. Gramm, L. Massüger, L. B. McCusker, Z. He, S. Hovmöller and X. Zou, Science 315, 1113 (2007).

[4] D. Shorokhov and A. H. Zewail, Phys. Chem. Chem. Phys. 10, 2879 (2008).

[5] B. J. Siwick, J. R. Dwyer, R. E. Jordan, and R. J. D. Miller, Science 302, 1382 (2003).

[6] M. Eichberger, H. Schäfer, M. Krumova, M. Beyer, J. Demsar, H. Berger, G. Moriena, G. Sciaini, and R. J. D. Miller, Nature (London) 468, 799 (2010).

[7] D. E. Sayers, E. A. Stern, and F. W. Lytle, Phys. Rev. Lett. 27, 1204 (1971).

[8] R. F. Pettifer, O. Mathon, S. Pascarelli, M. D. Cooke, and M. R. J. Gibbs, Nature (London) 435, 78 (2005).

[9] P. Glatzel and U. Bergmann, Coord. Chem. Rev. 249, 65 (2005).
[10] C. Bressler and M. Chergui, Chem. Rev. 104, 1781 (2004).

[11] S. A. Chambers, Adv. Phys. 40, 347 (1991).

[12] Ph. Hofmann, K.-M. Schindler, S. Bao, A. M. Bradshaw, and D. P. Woodruff, Nature (London) 368, 131 (1994).

[13] D. P. Woodruff, Surf. Sci. Rep. 62, 1 (2007).

[14] H. N. Chapman and K. A. Nugent, Nat. Photon. 4, 833 (2010).

[15] A. Cho, Science 330, 1470 (2010).

[16] H. N. Chapman et al., Nature (London) 470, 73 (2011).

[17] M. M. Seibert et al., Nature (London) 470, 78 (2011).

[18] F. Krasniqi, B. Najjari, L. Struder, D. Rolles, A. Voitkiv, and J. Ullrich, Phys. Rev. A 81, 033411 (2010).

[19] C. Miron and P. Morin, in Handbook of High-Resolution Spectroscopy, edited by M. Quack and F. Merkt (John Wiley \& Sons, Chichester, UK, 2011), p. 1655.

[20] S. E. Canton, E. Plésiat, J. D. Bozek, B. S. Rude, P. Decleva, and F. Martín, Proc. Natl. Acad. Sci. USA 108, 7302 (2011).

[21] X.-J. Liu, N. A. Cherepkov, S. K. Semenov, V. Kimberg, F. Gel'mukhanov, G. Prümper, T. Lischke, 
T. Tanaka, M. Hoshino, H. Tanaka, and K. Ueda, J. Phys. B 39, 4801 (2006).

[22] A. L. Landers, Th. Weber, I. Ali, A. Cassimi, M. Hattass, O. Jagutzki, A. Nauert, T. Osipov, A. Staudte, M. H. Prior, H. S.-Böcking, C. L. Cocke, and R. Dörner, Phys. Rev. Lett. 87, 013002 (2001).

[23] B. Zimmermann et al., Nat. Phys. 4, 649 (2008).

[24] M. Meckel, D. Comtois, D. Zeidler, A. Staudte, D. Pavicic, H. C. Bandulet, H. Pépin, J. C. Kieffer, R. Dörner, D. M. Villeneuve, and P. B. Corkum, Science 320, 1478 (2008).

[25] M. Okunishi, H. Niikura, R. R. Lucchese, T. Morishita, and K. Ueda, Phys. Rev. Lett. 106, 063001 (2011).

[26] T. X. Carroll, N. Berrah, J. Bozek, J. Hahne, E. Kukk, L. J. Saethre, and T. D. Thomas, Phys. Rev. A 59, 3386 (1999).

[27] U. Hergenhahn, J. Phys. B 37, R89 (2004).

[28] E. Kukk, K. Ueda, U. Hergenhahn, X.-J. Liu, G. Prümper, H. Yoshida, Y. Tamenori, C. Makochekanwa, T. Tanaka, M. Kitajima, and H. Tanaka, Phys. Rev. Lett. 95, 133001 (2005).

[29] E. Kukk, T. D. Thomas, and K. Ueda, J. Electron Spectrosc. Relat. Phenom. 183, 53 (2011).

[30] H. Bachau, E. Cormier, P. Decleva, J. E. Hansen, and F. Martín, Rep. Prog. Phys. 64, 1815 (2001).

[31] M. Venuti, M. Stener, and P. Decleva, Chem. Phys. 234, 95 (1998).

[32] T. Karlsen and K.-J. Borve, J. Chem. Phys. 112, 7986 (2000); Sons, Inc, 1975).

[33] C. Miron, [http://www.synchrotron-soleil.fr/Recherche/ LignesLumiere/PLEIADES].
[34] O. Travnikova, J.-C. Liu, A. Lindblad, C. Nicolas, J. Söderström, V. Kimberg, F. Gel'mukhanov, and C. Miron, Phys. Rev. Lett. 105, 233001 (2010).

[35] J. Södreström, A. Lindblad, A. Grum-Grzhimailo, O. Travnikova, C. Nicolas, S. Svensson, and C. Miron, New J. Phys. 13, 073014 (2011).

[36] C. Miron, C. Nicolas, O. Travnikova, P. Morin, Y. Sun, F. Gelmukhanov, N. Kosugi, and V. Kimberg, Nat. Phys. 8, 135 (2012).

[37] Y.-P. Sun, C.-K. Wang, and F. Gel'mukhanov, Phys. Rev. A 82, 052506 (2010).

[38] T. D. Thomas, E. Kukk, K. Ueda, T. Ouchi, K. Sakai, T. X. Carroll, C. Nicolas, O. Travnikova, and C. Miron, Phys. Rev. Lett. 106, 193009 (2011).

[39] E. Kukk, Curve fitting macro package SPANCF [http:// www.physics.utu.fi/en/research/material_science/Fitting.html]

[40] T. D. Thomas, E. Kukk, R. Sankari, H. Fukuzawa, G. Prümper, K. Ueda, R. Püttner, J. Harries, Y. Tamenori, T. Tanaka, M. Hoshino, and H. Tanaka, J. Chem. Phys. 128, 144311 (2008).

[41] P. Van der Straten, R. Morgenstern, and A. Niehaus, Z. Phys. D 8, 35 (1988).

[42] H.-M. Köppe, B.-S. Itchkawitz, A.-L.-D. Kilcoyne, J. Feldhaus, B. Kempgens, A. Kivimäki, M. Neeb, and A.-M. Bradshaw, Phys. Rev. A 53, 4120 (1996).

[43] M. Abramowitz and I. A. Stegun, Handbook of Mathematical Functions with Formulas, Graphs, and Mathematical Tables (Dover, New York 1964).

[44] L. Argenti, E. Plésiat, P. Decleva, and F. Martín (to be published).

[45] R. Georges, M. Herman, J.-C. Hilico, and O. Robert, J. Mol. Spectrosc. 187, 13 (1998). 